\title{
Microstructures and Properties of As-Cast AlCrFeMnV, AlCrFeTiV, and AlCrMnTiV High Entropy Alloys
}

\author{
Keith E. Knipling ${ }^{1}$, Prithvi U. Narayana ${ }^{2}$ and Lily T. Nguyen ${ }^{1}$ \\ 1. U. S. Naval Research Laboratory, Multifunctional Materials Branch, Washington, DC \\ 2. Thomas Jefferson High School for Science and Technology, Alexandria, VA
}

High entropy alloys (HEAs) typically contain five or more principal elements in nearly equiatomic proportions. In this new approach to alloy design, HEAs can be formed from a virtually limitless combination of elements. A strategy for accelerated discovery and optimization of possible HEA combinations has recently been developed by Senkov et al [1], who used thermodynamic modeling to calculate the equilibrium phases and properties of thousands of HEA combinations. The present study is an experimental verification of these predictions on three selected equimolar alloys: $\mathrm{AlCrFeMnV}$, AlCrFeTiV, and AlCrMnTiV, which were chosen as potential replacements for titanium alloys in extreme and corrosive environments. We report on their as-cast microstructures as measured by scanning electron microscopy (SEM), electron backscattered diffraction (EBSD), and atom probe tomography (APT).

Figure 1 shows the as-cast microstructures of the alloys, as measured by EBSD. AlCrFeMnV and AlCrMnTiV are a single-phase BCC solid solution while AlCrFeTiV, which is also predominantly BCC , also has an additional minor HCP phase. These observations are consistent with thermodynamic calculations [1], which predict that AlCrFeMnV and AlCrMnTiV would form BCC solid solutions at ambient temperature, while AlCrFeTiV would have two phases: BCC and a HCP C14 Laves phase. The structure of the C14 Laves phase has been confirmed by X-ray diffraction.

The two-phase microstructure of AlCrFeTiV is displayed more clearly in Figure 2. The HCP phase has a star shaped morphology that is present both on the grain boundaries (intergranular) and in the grains (intragranular). Figure 2(b), an IPF map showing the crystallographic orientations of the BCC matrix, shows that the Laves phase forms on multiple BCC grain boundaries and in multiple grains. Figure 2(c) is an IPF map showing the crystallographic orientations of the hexagonal C14 Laves phase itself, demonstrating that the Laves phase has multiple orientations.

Energy dispersive spectroscopy (EDS) was used to map the chemical segregation between the BCC and HCP phases in the AlCrFeTiV alloy. These maps, displayed in Figure 3, indicate that the BCC phase in $\mathrm{AlCrFeTiV}$ is enriched in $\mathrm{Fe}, \mathrm{Ti}$, and (to a lesser extent) $\mathrm{Al}$, while the $\mathrm{HCP}$ phase is enriched in $\mathrm{Cr}$ and V. The APT reconstruction in Figure 3 displays the position of each atom in this alloy in three dimensions, providing a more accurate measure of the compositions of the BCC and HCP phases. The partitioning of atoms between these two phases is quantified in the inset proximity histogram [2] [3].

References:

[1] ON Senkov et al, CALPHAD 50 (2015), p. 32.

[2] O Hellman et al, Microscopy and Microanalysis 6 (2000), p. 437.

[3] This work was funded by the U. S. Naval Research Laboratory under the auspices of the Office of

Naval Research. We thank Dan Miracle (Air Force Research Laboratory) for stimulating discussions. 

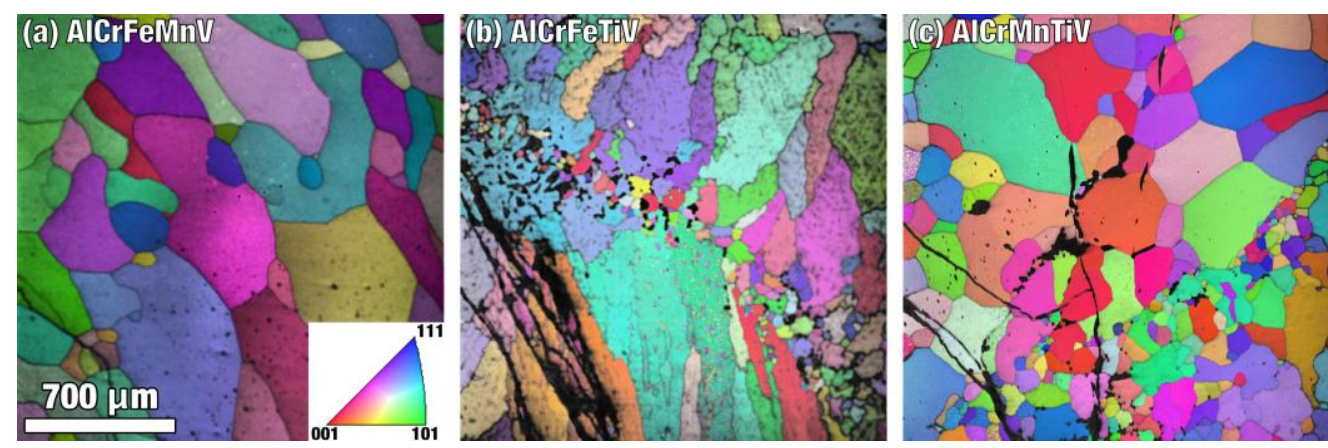

Figure 1. Inverse pole figure maps of the as-cast alloys: (a) $\mathrm{AlCrFeMnV}$, (b) $\mathrm{AlCrFeTiV}$, and (c) AlCrMnTiV.
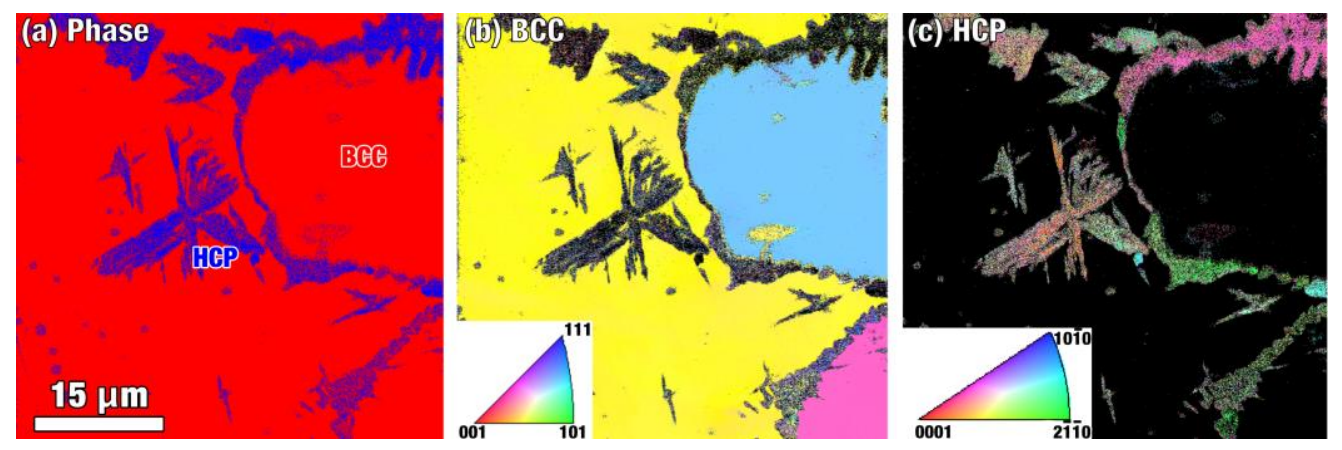

Figure 2. (a) Phase map displaying BCC (red) and HCP (blue) constituent phases. (b) Inverse pole figure map of the BCC phase. (c) Inverse pole figure map of the HCP phase.
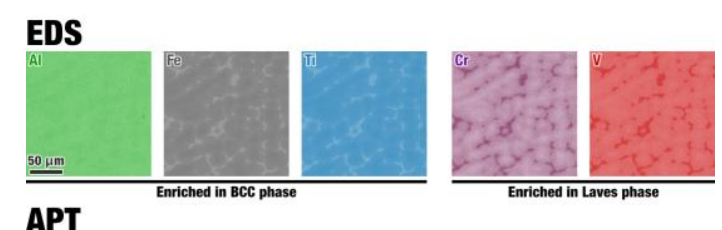

APT
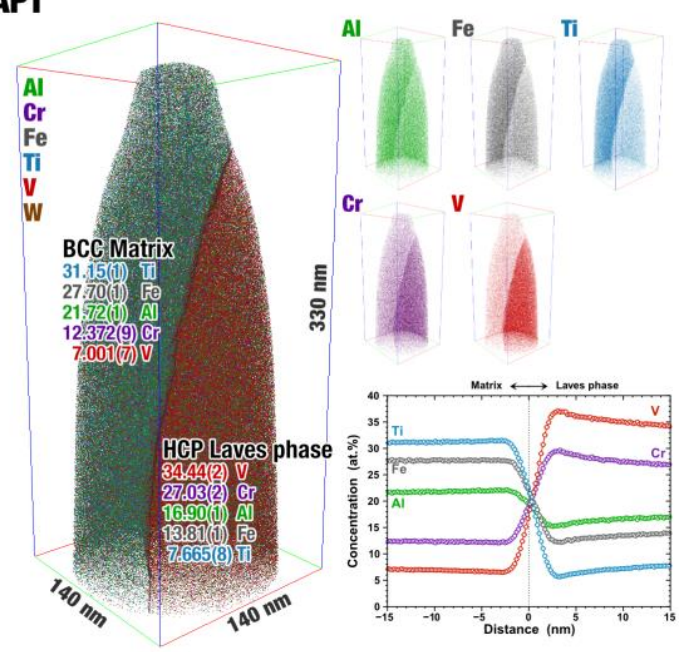

Figure 3. Energy dispersive spectroscopy (EDS) maps and atom probe tomography (APT) reconstructions displaying the chemical segregation between the $\mathrm{BCC}$ and $\mathrm{HCP}$ phases in $\mathrm{AlCrFeTiV}$. 